\title{
Estimates for singular integrals and extrapolation
}

by

\author{
Shuichi SATo (Kanazawa)
}

\begin{abstract}
We study singular integrals with rough kernels, which belong to a class of singular Radon transforms. We prove certain estimates for the singular integrals that are useful in an extrapolation argument. As an application, we prove $L^{p}$ boundedness of the singular integrals under a certain sharp size condition on their kernels.
\end{abstract}

1. Introduction. Let $\Omega$ be a function in $L^{1}\left(S^{n-1}\right)$ satisfying

$$
\int_{S^{n-1}} \Omega(\theta) d \sigma(\theta)=0
$$

where $d \sigma$ denotes the Lebesgue surface measure on the unit sphere $S^{n-1}$ in $\mathbb{R}^{n}$. In this note we assume $n \geq 2$. For $s \geq 1$, let $\Delta_{s}$ denote the collection of measurable functions $h$ on $\mathbb{R}_{+}=\{t \in \mathbb{R}: t>0\}$ satisfying

$$
\|h\|_{\Delta_{s}}=\sup _{j \in \mathbb{Z}}\left(\int_{2^{j}}^{2^{j+1}}|h(t)|^{s} d t / t\right)^{1 / s}<\infty,
$$

where $\mathbb{Z}$ denotes the set of integers. We note that $\Delta_{s} \subset \Delta_{t}$ if $s>t$. In this note we always assume $h \in \Delta_{1}$. Let $P(y)=\left(P_{1}(y), \ldots, P_{d}(y)\right)$ be a polynomial mapping, where each $P_{j}$ is a real-valued polynomial on $\mathbb{R}^{n}$. We consider a singular integral operator of the form

$$
\begin{aligned}
T(f)(x) & =\mathrm{p} \cdot \mathrm{v} \cdot \int_{\mathbb{R}^{n}} f(x-P(y)) K(y) d y \\
& =\lim _{\varepsilon \rightarrow 0} \int_{|y|>\varepsilon} f(x-P(y)) K(y) d y,
\end{aligned}
$$

for an appropriate function $f$ on $\mathbb{R}^{d}$, where $K(y)=h(|y|) \Omega\left(y^{\prime}\right)|y|^{-n}, y^{\prime}=$ $|y|^{-1} y$. Then $T(f)$ belongs to a class of singular Radon transforms. See Stein [20], Fan-Pan [8] and Al-Salman-Pan [1] for this singular integral.

2000 Mathematics Subject Classification: Primary 42B20, 42B25.

Key words and phrases: singular integrals, singular Radon transforms, maximal functions, extrapolation. 
When $h=1$ (a constant function), $n=d$ and $P(y)=y$, we also write $T(f)=S(f)$. Let $\hat{f}(\xi)=\int_{\mathbb{R}^{d}} f(x) e^{-2 \pi i\langle x, \xi\rangle} d x$ be the Fourier transform of $f$, where $\langle\cdot, \cdot\rangle$ denotes the inner product in $\mathbb{R}^{d}$. Then it is known that $(S f)^{\wedge}(\xi)=m\left(\xi^{\prime}\right) \hat{f}(\xi)$, where

$$
m\left(\xi^{\prime}\right)=-\int_{S^{n-1}} \Omega(\theta)\left[i \frac{\pi}{2} \operatorname{sgn}\left(\left\langle\xi^{\prime}, \theta\right\rangle\right)+\log \left|\left\langle\xi^{\prime}, \theta\right\rangle\right|\right] d \sigma(\theta) .
$$

Using this, we can show that $S$ extends to a bounded operator on $L^{2}$ if $\Omega \in L \log L\left(S^{n-1}\right)$, where $L \log L\left(S^{n-1}\right)$ denotes the Zygmund class of all those functions $\Omega$ on $S^{n-1}$ which satisfy

$$
\int_{S^{n-1}}|\Omega(\theta)| \log (2+|\Omega(\theta)|) d \sigma(\theta)<\infty .
$$

Furthermore, if $\Omega \in L \log L\left(S^{n-1}\right)$, by the method of rotations of CalderónZygmund (see [2]) it can be shown that $S$ extends to a bounded operator on $L^{p}$ for all $p \in(1, \infty)$.

When $n=d$ and $P(y)=y$, R. Fefferman [10] proved that if $h$ is bounded and $\Omega$ satisfies a Lipschitz condition of positive order on $S^{n-1}$, then the singular integral operator $T$ in (1.2) is bounded on $L^{p}$ for $1<p<\infty$. Namazi [13] improved this result by replacing the Lipschitz condition by the condition that $\Omega \in L^{q}\left(S^{n-1}\right)$ for some $q>1$. In [7], Duoandikoetxea and Rubio de Francia developed methods which can be used to study mapping properties of several kinds of operators in harmonic analysis including the singular integrals considered in [13]. Also, see $[6,25]$ for weighted $L^{p}$ boundedness of singular integrals, and $[21,22]$ for background materials.

For the rest of this note we assume that the polynomial mapping $P$ in (1.2) satisfies $P(-y)=-P(y)$ and $P \neq 0$. We shall prove the following result:

Theorem 1. Let $\Omega \in L^{q}\left(S^{n-1}\right), q \in(1,2]$ and $h \in \Delta_{s}, s \in(1,2]$. Suppose $\Omega$ satisfies (1.1). Let $T$ be as in (1.2). Then

$$
\|T(f)\|_{L^{p}\left(\mathbb{R}^{d}\right)} \leq C_{p}(q-1)^{-1}(s-1)^{-1}\|\Omega\|_{L^{q}\left(S^{n-1}\right)}\|h\|_{\Delta_{s}}\|f\|_{L^{p}\left(\mathbb{R}^{d}\right)}
$$

for all $p \in(1, \infty)$, where the constant $C_{p}$ is independent of $q, s, \Omega$ and $h$. Also, the constant $C_{p}$ is independent of the polynomials $P_{j}$ if we fix $\operatorname{deg}\left(P_{j}\right)$ $(j=1, \ldots, d)$.

See $[15,16,17]$ for relevant results. In Al-Salman-Pan [1], $L^{p}$ boundedness of $T$ was proved under the condition that $\Omega$ is a function in $L \log L\left(S^{n-1}\right)$ satisfying (1.1) and $h \in \Delta_{s}$ for some $s>1$ ([1, Theorem 1.3]). Also it is noted there that estimates like those in Theorem 1 (with $s$ being fixed) can be used to prove the same result by applying an extrapolation method, but such estimates are yet to be proved (see [1, p. 156]). In [1], 
the authors also considered singular integrals defined by certain polynomial mappings $P$ which do not satisfy the condition $P(-y)=-P(y)$.

As a consequence of Theorem 1 we can give a different proof of $[1$, Theorem 1.3] via an extrapolation method; in fact, we can get an improved result. For a positive number $a$ and a function $h$ on $\mathbb{R}_{+}$, let

$$
L_{a}(h)=\sup _{j \in \mathbb{Z}} \int_{2^{j}}^{2^{j+1}}|h(r)|(\log (2+|h(r)|))^{a} d r / r .
$$

We define a class $\mathcal{L}_{a}$ to be the space of all those measurable functions $h$ on $\mathbb{R}_{+}$which satisfy $L_{a}(h)<\infty$. Also, let

$$
N_{a}(h)=\sum_{m \geq 1} m^{a} 2^{m} d_{m}(h),
$$

where $d_{m}(h)=\sup _{k \in \mathbb{Z}} 2^{-k}|E(k, m)|$ with $E(k, m)=\left\{r \in\left(2^{k}, 2^{k+1}\right]: 2^{m-1}\right.$ $\left.<|h(r)| \leq 2^{m}\right\}$ for $m \geq 2, E(k, 1)=\left\{r \in\left(2^{k}, 2^{k+1}\right]: 0<|h(r)| \leq 2\right\}$. We denote by $\mathcal{N}_{a}$ the class of all those measurable functions $h$ on $\mathbb{R}_{+}$such that $N_{a}(h)<\infty$. Then we readily see that $N_{a}(h)<\infty$ implies $L_{a}(h)<\infty$. Conversely, if $L_{a+b}(h)<\infty$ for some $b>1$, then $N_{a}(h)<\infty$. To see this, note that

$$
2^{m} m^{a+b} 2^{-k}|E(k, m)| \leq C \int_{E(k, m)}|h(r)|(\log (2+|h(r)|))^{a+b} d r / r \leq C L_{a+b}(h)
$$

for $m \geq 2$; thus $N_{a}(h) \leq 2 d_{1}(h)+C L_{a+b}(h) \sum_{m>2} m^{-b}<\infty$. By Theorem 1 and an extrapolation method we have the following:

THEOREM 2. Suppose $\Omega$ is a function in $L \log L\left(S^{n-1}\right)$ satisfying (1.1) and $h \in \mathcal{N}_{1}$. Let $T$ be as in (1.2). Then

$$
\|T(f)\|_{L^{p}\left(\mathbb{R}^{d}\right)} \leq C_{p}\|f\|_{L^{p}\left(\mathbb{R}^{d}\right)}
$$

for all $p \in(1, \infty)$, where $C_{p}$ is independent of the polynomials $P_{j}$ if the polynomials are of fixed degree.

By Theorem 2 and the remark preceding it we see that $T$ is bounded on $L^{p}$ for all $p \in(1, \infty)$ if $\Omega$ is as in Theorem 2 and $h \in \mathcal{L}_{a}$ for some $a>2$. When $n=d, P(y)=y, \Omega$ is as in Theorem 2 and $h$ is a constant function, it is known that $T$ is of weak type $(1,1)$; see $[5,18,4,9,11,12,19,23,24]$.

In Section 2, we shall prove Theorem 1. Applying the methods of [7] involving the Littlewood-Paley theory and using results of $[8,14]$, we shall prove $L^{p}$ estimates for certain maximal and singular integral operators related to the operator $T$ in Theorem 1 (Lemmas 1 and 2). Lemma 1 is used to prove Lemma 2 . By Lemma 2 we can easily prove Theorem 1 . A key idea of the proof of Theorem 1 is to apply a Littlewood-Paley decomposition adapted to a suitable lacunary sequence depending on $q$ and $s$ for which 
$\Omega \in L^{q}\left(S^{n-1}\right)$ and $h \in \Delta_{s}$. The method of appropriately choosing a lacunary sequence was inspired by [1], where, in a somewhat different way from ours, a similar method was used to study several classes of singular integrals.

We shall prove Theorem 2 in Section 3. Finally, in Section 4, we consider the maximal operator

$$
T^{*}(f)(x)=\sup _{N, \varepsilon>0}\left|\int_{\varepsilon<|y|<N} f(x-P(y)) K(y) d y\right|,
$$

where $P$ and $K$ are as in (1.2). We shall prove analogs of Theorems 1 and 2 for the operator $T^{*}$.

Throughout this note, the letter $C$ will be used to denote non-negative constants which may be different in different occurrences.

2. Proof of Theorem 1. Let $\Omega, h$ be as in Theorem 1 . We consider the singular integral $T(f)$ defined in (1.2). Let $\varrho \geq 2$ and $E_{k}=\left\{x \in \mathbb{R}^{n}\right.$ : $\left.\varrho^{k}<|x| \leq \varrho^{k+1}\right\}$. Then $T(f)(x)=\sum_{-\infty}^{\infty} \sigma_{k} * f(x)$, where $\left\{\sigma_{k}\right\}$ is a sequence of Borel measures on $\mathbb{R}^{d}$ such that

$$
\sigma_{k} * f(x)=\int_{E_{k}} f(x-P(y)) K(y) d y .
$$

We note that

$$
\left(\sigma_{k} * f\right)^{\wedge}(\xi)=\hat{f}(\xi) \int_{E_{k}} e^{-2 \pi i\langle P(y), \xi\rangle} K(y) d y
$$

We write

$$
P(y)=\sum_{j=1}^{\ell} Q_{j}(y), \quad Q_{j}(y)=\sum_{|\gamma|=N(j)} a_{\gamma} y^{\gamma} \quad\left(a_{\gamma} \in \mathbb{R}^{d}\right),
$$

where $Q_{j} \neq 0,1 \leq N(1)<\cdots<N(\ell), \gamma=\left(\gamma_{1}, \ldots, \gamma_{n}\right)$ is a multi-index, $y^{\gamma}=y_{1}^{\gamma_{1}} \ldots y_{n}^{\gamma_{n}}$ and $|\gamma|=\gamma_{1}+\cdots+\gamma_{n}$. We set $\beta_{m}=\varrho^{N(m)}$ and $\alpha_{m}=$ $(q-1)(s-1) /(2 q s N(m))$ for $1 \leq m \leq \ell$. Put $P^{(m)}(y)=\sum_{j=1}^{m} Q_{j}(y)$ and define a sequence $\mu^{(m)}=\left\{\mu_{k}^{(m)}\right\}$ of positive measures on $\mathbb{R}^{d}$ by

$$
\mu_{k}^{(m)} * f(x)=\int_{E_{k}} f\left(x-P^{(m)}(y)\right)|K(y)| d y
$$

for $m=1, \ldots, \ell$. Also, define $\mu^{(0)}=\left\{\mu_{k}^{(0)}\right\}$ by $\mu_{k}^{(0)}=\left(\int_{E_{k}}|K(y)| d y\right) \delta$, where $\delta$ is Dirac's delta function on $\mathbb{R}^{d}$. For a sequence $\nu=\left\{\nu_{k}\right\}$ of finite Borel measures on $\mathbb{R}^{d}$, we define the maximal operator $\nu^{*}$ by $\nu^{*}(f)(x)=$ $\sup _{k}|| \nu_{k}|* f(x)|$, where $\left|\nu_{k}\right|$ denotes the total variation. We consider the maximal operators $\left(\mu^{(m)}\right)^{*}(0 \leq m \leq \ell)$. We also write $\left(\mu^{(\ell)}\right)^{*}=\mu_{\varrho}^{*}$.

Let

$$
L_{j}(\xi)=\left(\left\langle a_{\gamma(j, 1)}, \xi\right\rangle, \ldots,\left\langle a_{\gamma\left(j, r_{j}\right)}, \xi\right\rangle\right),
$$


where $\{\gamma(j, k)\}_{k=1}^{r_{j}}$ is an enumeration of $\{\gamma\}_{|\gamma|=N(j)}$ for $1 \leq j \leq \ell$. Then $L_{j}$ is a linear mapping from $\mathbb{R}^{d}$ to $\mathbb{R}^{r_{j}}$. Let $s_{j}=\operatorname{rank} L_{j}$. There exist non-singular linear transformations $R_{j}: \mathbb{R}^{d} \rightarrow \mathbb{R}^{d}$ and $H_{j}: \mathbb{R}^{s_{j}} \rightarrow \mathbb{R}^{s_{j}}$ such that

$$
\left|H_{j} \pi_{s_{j}}^{d} R_{j}(\xi)\right| \leq\left|L_{j}(\xi)\right| \leq C\left|H_{j} \pi_{s_{j}}^{d} R_{j}(\xi)\right|,
$$

where $\pi_{s_{j}}^{d}(\xi)=\left(\xi_{1}, \ldots, \xi_{s_{j}}\right)$ is the projection and $C$ depends only on $r_{j}$ (a proof can be found in [8]). Let $\left\{\sigma_{k}^{(m)}\right\}(0 \leq m \leq \ell)$ be a sequence of Borel measures on $\mathbb{R}^{d}$ such that

$$
\sigma_{k}^{(m)} * f(x)=\int_{E_{k}} f\left(x-P^{(m)}(y)\right) K(y) d y
$$

for $m=1, \ldots, \ell$, while $\sigma_{k}^{(0)}=0$. Let $\varphi \in C_{0}^{\infty}(\mathbb{R})$ be supported in $\{|r| \leq 1\}$ and $\varphi(r)=1$ for $|r|<1 / 2$. Define a sequence $\tau^{(m)}=\left\{\tau_{k}^{(m)}\right\}$ of Borel measures by

$$
\hat{\tau}_{k}^{(m)}(\xi)=\hat{\sigma}_{k}^{(m)}(\xi) \Phi_{k, m}(\xi)-\hat{\sigma}_{k}^{(m-1)}(\xi) \Phi_{k, m-1}(\xi)
$$

for $m=1, \ldots, \ell$, where

$$
\Phi_{k, m}(\xi)=\prod_{j=m+1}^{\ell} \varphi\left(\beta_{j}^{k}\left|H_{j} \pi_{s_{j}}^{d} R_{j}(\xi)\right|\right)
$$

if $0 \leq m \leq \ell-1$ and $\Phi_{k, \ell}=1$. Then $\sigma_{k}=\sigma_{k}^{(\ell)}=\sum_{m=1}^{\ell} \tau_{k}^{(m)}$. We note that

$$
\Phi_{k, m}(\xi) \varphi\left(\beta_{m}^{k}\left|H_{m} \pi_{s_{m}}^{d} R_{m}(\xi)\right|\right)=\Phi_{k, m-1}(\xi) \quad(1 \leq m \leq \ell) .
$$

For $1 \leq m \leq \ell$, let $T_{\varrho}^{(m)}(f)=\sum_{k} \tau_{k}^{(m)} * f$. Then $T=\sum_{m=1}^{\ell} T_{\varrho}^{(m)}$.

For $p \in(1, \infty)$ we put $p^{\prime}=p /(p-1)$ and $\delta(p)=\left|1 / p-1 / p^{\prime}\right|$. Let $\theta \in(0,1)$. Then we have the following $L^{p}$ estimates for $\left(\mu^{(m)}\right)^{*}$ and $T_{\varrho}^{(m)}$.

Lemma 1. For $p>1+\theta$ and $0 \leq j \leq \ell$, we have

$$
\begin{aligned}
& \left\|\left(\mu^{(j)}\right)^{*}(f)\right\|_{L^{p}\left(\mathbb{R}^{d}\right)} \\
& \quad \leq C(\log \varrho)\|\Omega\|_{L^{q}\left(S^{n-1}\right)}\|h\|_{\Delta_{s}}\left(1-\varrho^{-\theta /\left(2 q^{\prime} s^{\prime}\right)}\right)^{-2 / p}\|f\|_{L^{p}\left(\mathbb{R}^{d}\right)} .
\end{aligned}
$$

Lemma 2. For $p \in(1+\theta,(1+\theta) / \theta)$ and $1 \leq m \leq \ell$, we have

$$
\begin{aligned}
& \left\|T_{\varrho}^{(m)}(f)\right\|_{L^{p}\left(\mathbb{R}^{d}\right)} \\
& \quad \leq C(\log \varrho)\|\Omega\|_{L^{q}\left(S^{n-1}\right)}\|h\|_{\Delta_{s}}\left(1-\varrho^{-\theta /\left(2 q^{\prime} s^{\prime}\right)}\right)^{-1-\delta(p)}\|f\|_{L^{p}\left(\mathbb{R}^{d}\right)} .
\end{aligned}
$$

The constants $C$ in Lemmas 1 and 2 are independent of $q, s \in(1,2], \Omega \in$ $L^{q}\left(S^{n-1}\right), h \in \Delta_{s}, \varrho$ and the coefficients of the polynomials $P_{k}(1 \leq k \leq d)$.

We prove Lemma 2 first, taking Lemma 1 for granted for the moment. Let $A=(\log \varrho)\|\Omega\|_{L^{q}\left(S^{n-1}\right)}\|h\|_{\Delta_{s}}$ and $B=\left(1-\beta_{m}^{-\theta \alpha_{m}}\right)^{-1}=\left(1-\varrho^{-\theta /\left(2 q^{\prime} s^{\prime}\right)}\right)^{-1}$. 
Then we have the following estimates:

$$
\begin{aligned}
\left\|\tau_{k}^{(m)}\right\| & \leq c_{1} A \quad\left(\left\|\tau_{k}^{(m)}\right\|=\left|\tau_{k}^{(m)}\right|\left(\mathbb{R}^{d}\right)\right), \\
\left|\hat{\tau}_{k}^{(m)}(\xi)\right| & \leq c_{2} A\left(\beta_{m}^{k}\left|L_{m}(\xi)\right|\right)^{-\alpha_{m}}, \\
\left|\hat{\tau}_{k}^{(m)}(\xi)\right| & \leq c_{3} A\left(\beta_{m}^{k+1}\left|L_{m}(\xi)\right|\right)^{\alpha_{m}}, \\
\left\|\left(\tau^{(m)}\right)^{*}(f)\right\|_{p} & \leq C_{p} A B^{2 / p}\|f\|_{p} \quad \text { for } p>1+\theta,
\end{aligned}
$$

for some constants $c_{i}(1 \leq i \leq 3)$ and $C_{p}$, where we simply write $\|f\|_{L^{p}\left(\mathbb{R}^{d}\right)}=$ $\|f\|_{p}$.

Now we prove the estimates (2.4)-(2.7). First we see that

$$
\begin{aligned}
\left\|\tau_{k}^{(m)}\right\| & \leq C\left(\left\|\sigma_{k}^{(m)}\right\|+\left\|\sigma_{k}^{(m-1)}\right\|\right) \\
& \leq C\|\Omega\|_{1} \int_{\varrho^{k}}^{\varrho^{k+1}}|h(r)| d r / r \leq C(\log \varrho)\|\Omega\|_{1}\|h\|_{\Delta_{1}} .
\end{aligned}
$$

From this (2.4) follows. To prove (2.5), define

$$
F(r, \xi)=\int_{S^{n-1}} \Omega(\theta) \exp \left(-2 \pi i\left\langle\xi, P^{(m)}(r \theta)\right\rangle\right) d \sigma(\theta) .
$$

Then, via Hölder's inequality, for $s \in(1,2]$ we see that

$$
\begin{aligned}
\left|\hat{\sigma}_{k}^{(m)}(\xi)\right| & =\left|\int_{\varrho^{k}}^{\varrho^{k+1}} h(r) F(r, \xi) d r / r\right| \\
& \leq\left(\int_{\varrho^{k}}^{\varrho^{k+1}}|h(r)|^{s} d r / r\right)^{1 / s}\left(\int_{\varrho^{k}}^{\varrho^{k+1}}|F(r, \xi)|^{s^{\prime}} d r / r\right)^{1 / s^{\prime}} \\
& \leq C(\log \varrho)^{1 / s}\|h\|_{\Delta_{s}}\|\Omega\|_{1}^{\left(s^{\prime}-2\right) / s^{\prime}}\left(\int_{\varrho^{\varrho^{k+1}}}|F(r, \xi)|^{2} d r / r\right)^{1 / s^{\prime}} .
\end{aligned}
$$

We need the following estimates for the last integral:

Lemma 3. Let $1<q \leq 2$ and $\Omega \in L^{q}\left(S^{n-1}\right)$. Then there exists a constant $C>0$ independent of $q, \varrho, \Omega$ and the coefficients of the polynomial components of $P^{(m)}$ such that

$$
\int_{\varrho^{k}}^{\varrho^{k+1}}|F(r, \xi)|^{2} d r / r \leq C(\log \varrho)\left(\beta_{m}^{k}\left|L_{m}(\xi)\right|\right)^{-1 /\left(2 q^{\prime} N(m)\right)}\|\Omega\|_{q}^{2} .
$$

Proof. Take an integer $\nu$ such that $2^{\nu}<\varrho \leq 2^{\nu+1}$. By the proof of Proposition 5.1 of [8] we have 


$$
\begin{aligned}
\int_{\varrho^{k}}^{\varrho^{k+1}}|F(r, \xi)|^{2} d r / r & =\int_{1}^{\varrho}\left|F\left(\varrho^{k} r, \xi\right)\right|^{2} d r / r \leq \sum_{j=0}^{\nu} \int_{2^{j}}^{2^{j+1}}\left|F\left(\varrho^{k} r, \xi\right)\right|^{2} d r / r \\
& \leq \sum_{j=0}^{\nu} C\left(\int_{1}^{2}\left|F\left(2^{j} \varrho^{k} r, \xi\right)\right|^{q^{\prime}} d r / r\right)^{2 / q^{\prime}} \\
& \leq \sum_{j=0}^{\nu} C\left(2^{j N(m)} \varrho^{k N(m)}\left|L_{m}(\xi)\right|\right)^{-1 /\left(2 N(m) q^{\prime}\right)}\|\Omega\|_{q}^{2} \\
& \leq C(\log \varrho)\left(\varrho^{k N(m)}\left|L_{m}(\xi)\right|\right)^{-1 /\left(2 N(m) q^{\prime}\right)}\|\Omega\|_{q}^{2} .
\end{aligned}
$$

This completes the proof of Lemma 3.

By (2.9) and Lemma 3 we have $\left|\hat{\sigma}_{k}^{(m)}(\xi)\right| \leq C A\left(\beta_{m}^{k}\left|L_{m}(\xi)\right|\right)^{-\alpha_{m}}$. Also, we have $\left\|\sigma_{k}^{(m-1)}\right\| \leq C A$ by (2.8). We can prove the estimate (2.5) by using these estimates in the definition of $\tau_{k}^{(m)}$ in $(2.2)$ and by noting that $\varphi$ is compactly supported. Next, to prove (2.6), using (1.1) when $m=1$, we see that

$$
\begin{aligned}
\left|\hat{\tau}_{k}^{(m)}(\xi)\right| \leq & \left|\left(\hat{\sigma}_{k}^{(m)}(\xi)-\hat{\sigma}_{k}^{(m-1)}(\xi)\right) \Phi_{k, m}(\xi)\right| \\
& +\left|\left(\Phi_{k, m}(\xi)-\Phi_{k, m-1}(\xi)\right) \hat{\sigma}_{k}^{(m-1)}(\xi)\right| \\
\leq & C\|\Omega\|_{1} \beta_{m}^{k+1}\left|L_{m}(\xi)\right| \int_{\varrho^{k}}^{\varrho^{k+1}}|h(r)| d r / r+C\left\|\sigma_{k}^{(m-1)}\right\| \beta_{m}^{k}\left|L_{m}(\xi)\right| \\
\leq & C(\log \varrho)\|\Omega\|_{1}\|h\|_{\Delta_{1}} \beta_{m}^{k+1}\left|L_{m}(\xi)\right|,
\end{aligned}
$$

where to get the last inequality we have used (2.8). By this and (2.8), we have

$$
\left|\hat{\tau}_{k}^{(m)}(\xi)\right| \leq C(\log \varrho)\|\Omega\|_{1}\|h\|_{\Delta_{1}}\left(\beta_{m}^{k+1}\left|L_{m}(\xi)\right|\right)^{c}
$$

for all $c \in(0,1]$, which implies (2.6). Finally, the estimate (2.7) follows from Lemma 1 since

$$
\left\|\left(\tau^{(m)}\right)^{*}(f)\right\|_{p} \leq C\left\|\left(\mu^{(m)}\right)^{*}(|f|)\right\|_{p}+C\left\|\left(\mu^{(m-1)}\right)^{*}(|f|)\right\|_{p} \leq C A B^{2 / p}\|f\|_{p}
$$

for $p>1+\theta$, where the first inequality can be seen by a change of variables and a well-known result on maximal functions (see [8]).

Let $\left\{\psi_{k}\right\}_{k=-\infty}^{\infty}$ be a sequence of non-negative functions in $C^{\infty}((0, \infty))$ $\operatorname{such}$ that $\operatorname{supp}\left(\psi_{k}\right) \subset\left[\beta_{m}^{-k-1}, \beta_{m}^{-k+1}\right]$,

$$
\sum_{k} \psi_{k}(t)^{2}=1, \quad\left|(d / d t)^{j} \psi_{k}(t)\right| \leq c_{j} / t^{j} \quad(j=1,2, \ldots) \quad \text { for all } t>0,
$$

where the constants $c_{j}$ are independent of $\beta_{m}$ (this is feasible since $\beta_{m} \geq 2$ ). 
Define an operator $S_{k}$ by $\left(S_{k}(f)\right)^{\wedge}(\xi)=\psi_{k}\left(\left|H_{m} \pi_{s_{m}}^{d} R_{m}(\xi)\right|\right) \hat{f}(\xi)$ and let

$$
V_{j}^{(m)}(f)=\sum_{k=-\infty}^{\infty} S_{j+k}\left(\tau_{k}^{(m)} * S_{j+k}(f)\right) .
$$

Then by Plancherel's theorem and the estimates (2.4)-(2.6) we have

$$
\begin{aligned}
\left\|V_{j}^{(m)}(f)\right\|_{2}^{2} & \leq \sum_{k} C \int_{D(j+k)}\left|\hat{\tau}_{k}^{(m)}(\xi)\right|^{2}|\hat{f}(\xi)|^{2} d \xi \\
& \leq C A^{2} \min \left(1, \beta_{m}^{-2 \alpha_{m}(|j|-2)}\right) \sum_{k} \int_{D(j+k)}|\hat{f}(\xi)|^{2} d \xi \\
& \leq C A^{2} \min \left(1, \beta_{m}^{-2 \alpha_{m}(|j|-2)}\right)\|f\|_{2}^{2},
\end{aligned}
$$

where $D(k)=\left\{\beta_{m}^{-k-1} \leq\left|H_{m} \pi_{s_{m}}^{d} R_{m}(\xi)\right| \leq \beta_{m}^{-k+1}\right\}$.

Applying the proof of Lemma on [7, p. 544] and using the estimates (2.4) and (2.7), we can prove the following.

Lemma 4. Let $u \in(1+\theta, 2]$. Define a number $v$ by $1 / v-1 / 2=1 /(2 u)$. Then we have the vector-valued inequality

$$
\left\|\left(\sum\left|\tau_{k}^{(m)} * g_{k}\right|^{2}\right)^{1 / 2}\right\|_{v} \leq\left(c_{1} C_{u}\right)^{1 / 2} A B^{1 / u}\left\|\left(\sum\left|g_{k}\right|^{2}\right)^{1 / 2}\right\|_{v},
$$

where the constants $c_{1}$ and $C_{u}$ are as in (2.4) and (2.7), respectively.

By the Littlewood-Paley theory we have

$$
\begin{gathered}
\left\|V_{j}^{(m)}(f)\right\|_{p} \leq c_{p}\left\|\left(\sum_{k}\left|\tau_{k}^{(m)} * S_{j+k}(f)\right|^{2}\right)^{1 / 2}\right\|_{p} \\
\left\|\left(\sum_{k}\left|S_{k}(f)\right|^{2}\right)^{1 / 2}\right\|_{p} \leq c_{p}\|f\|_{p}
\end{gathered}
$$

where $1<p<\infty$ and $c_{p}$ is independent of $\beta_{m}$ and the linear transformations $R_{m}, H_{m}$. Suppose that $1+\theta<p \leq 4 /(3-\theta)$. Then we can find $u \in(1+\theta, 2]$ such that $1 / p=1 / 2+(1-\theta) /(2 u)$. Let $v$ be defined by $u$ as in Lemma 4 . Then by (2.11)-(2.13) we have

$$
\left\|V_{j}^{(m)}(f)\right\|_{v} \leq C A B^{1 / u}\|f\|_{v} .
$$

Since $1 / p=\theta / 2+(1-\theta) / v$, interpolating between (2.10) and (2.14), we have

$$
\left\|V_{j}^{(m)}(f)\right\|_{p} \leq C A B^{(1-\theta) / u} \min \left(1, \beta_{m}^{-\theta \alpha_{m}(|j|-2)}\right)\|f\|_{p} .
$$

It follows that

$$
\begin{aligned}
\left\|T_{\varrho}^{(m)}(f)\right\|_{p} & \leq \sum_{j}\left\|V_{j}^{(m)}(f)\right\|_{p} \leq C A B^{(1-\theta) / u}\left(1-\beta_{m}^{-\theta \alpha_{m}}\right)^{-1}\|f\|_{p} \\
& \leq C A B^{2 / p}\|f\|_{p}
\end{aligned}
$$


where we have used the inequality $\sum \min \left(1, \beta_{m}^{-\theta \alpha_{m}(|j|-2)}\right) \leq 5\left(1-\beta_{m}^{-\theta \alpha_{m}}\right)^{-1}$. We also have $\left\|T_{\varrho}^{(m)}(f)\right\|_{2} \leq \sum\left\|V_{j}^{(m)}(f)\right\|_{2} \leq C A B\|f\|_{2}$ by $(2.10)$, since $B \geq$ $\left(1-\beta_{m}^{-\alpha_{m}}\right)^{-1}$. By duality and interpolation, we can now get the conclusion of Lemma 2.

Next, we give a proof of Lemma 1 . We argue by induction on $j$. So assuming (2.3) holds for $j=m-1,1 \leq m \leq \ell$, we will prove it for $j=m$. Let $\varphi \in C_{0}^{\infty}(\mathbb{R})$ be as above. Define a sequence $\eta^{(m)}=\left\{\eta_{k}^{(m)}\right\}$ of Borel measures on $\mathbb{R}^{d}$ by

$$
\hat{\eta}_{k}^{(m)}(\xi)=\varphi\left(\beta_{m}^{k}\left|H_{m} \pi_{s_{m}}^{d} R_{m}(\xi)\right|\right) \hat{\mu}_{k}^{(m-1)}(\xi) .
$$

Then by (2.3) with $j=m-1$, we have

$$
\left\|\left(\eta^{(m)}\right)^{*}(f)\right\|_{p} \leq C\left\|\left(\mu^{(m-1)}\right)^{*}(f)\right\|_{p} \leq C A B^{2 / p}\|f\|_{p}
$$

for $p>1+\theta$. Furthermore, we have the following:

$$
\begin{gathered}
\left\|\eta_{k}^{(m)}\right\|+\left\|\mu_{k}^{(m)}\right\| \leq C\left\|\mu_{k}^{(m-1)}\right\|+\left\|\mu_{k}^{(m)}\right\| \leq C\|\Omega\|_{1} \int_{\varrho^{k}}^{\varrho^{k+1}}|h(r)| d r / r \\
\leq C(\log \varrho)\|\Omega\|_{1}\|h\|_{\Delta_{1}} \leq C A, \\
\left|\hat{\mu}_{k}^{(m)}(\xi)-\hat{\eta}_{k}^{(m)}(\xi)\right| \leq C(\log \varrho)\|\Omega\|_{1}\|h\|_{\Delta_{1}}\left(\beta_{m}^{k+1}\left|L_{m}(\xi)\right|\right)^{\alpha_{m}} \\
\leq C A\left(\beta_{m}^{k+1}\left|L_{m}(\xi)\right|\right)^{\alpha_{m}} \\
\left|\hat{\mu}_{k}^{(m)}(\xi)\right| \leq C A\left(\beta_{m}^{k}\left|L_{m}(\xi)\right|\right)^{-\alpha_{m}} \\
\left|\hat{\eta}_{k}^{(m)}(\xi)\right| \leq C(\log \varrho)\|h\|_{\Delta_{1}}\|\Omega\|_{1}\left(\beta_{m}^{k}\left|L_{m}(\xi)\right|\right)^{-\alpha_{m}} \\
\leq C A\left(\beta_{m}^{k}\left|L_{m}(\xi)\right|\right)^{-\alpha_{m}}
\end{gathered}
$$

To see $(2.18)$ we note that

$$
\begin{aligned}
& \left|\hat{\mu}_{k}^{(m)}(\xi)-\hat{\eta}_{k}^{(m)}(\xi)\right| \\
& \quad \leq\left|\hat{\mu}_{k}^{(m)}(\xi)-\hat{\mu}_{k}^{(m-1)}(\xi)\right|+\left|\left(\varphi\left(\beta_{m}^{k}\left|H_{m} \pi_{s_{m}}^{d} R_{m}(\xi)\right|\right)-1\right) \hat{\mu}_{k}^{(m-1)}(\xi)\right| .
\end{aligned}
$$

Thus arguing as in the proof of (2.6), we have the first inequality of (2.18). The estimate (2.19) follows from the arguments used to prove (2.5). Also, the first inequality of $(2.20)$ is a consequence of the definition of $\eta_{k}^{(m)}$ and (2.17).

Since $\left\|\left(\mu^{(m)}\right)^{*}(f)\right\|_{\infty} \leq C A\|f\|_{\infty}$, by interpolation, it suffices to prove (2.3) with $j=m$ for $p \in(1+\theta, 2]$. Define a sequence $\nu^{(m)}=\left\{\nu_{k}^{(m)}\right\}$ of Borel measures by $\nu_{k}^{(m)}=\mu_{k}^{(m)}-\eta_{k}^{(m)}$. Let

Then

$$
g_{m}(f)(x)=\left(\sum\left|\nu_{k}^{(m)} * f(x)\right|^{2}\right)^{1 / 2} \text {. }
$$

$$
\left(\mu^{(m)}\right)^{*}(f) \leq g_{m}(f)+\left(\eta^{(m)}\right)^{*}(|f|)
$$


Thus, by (2.16), to get (2.3) with $j=m$ it suffices to prove $\left\|g_{m}(f)\right\|_{p} \leq$ $C A B^{2 / p}\|f\|_{p}$ for $p \in(1+\theta, 2]$ with an appropriate constant $C$. By a wellknown property of Rademacher's functions, this follows from

$$
\left\|U_{\varepsilon}^{(m)}(f)\right\|_{p} \leq C A B^{2 / p}\|f\|_{p}
$$

for $p \in(1+\theta, 2]$, where $U_{\varepsilon}^{(m)}(f)=\sum_{k} \varepsilon_{k} \nu_{k}^{(m)} * f$ with $\varepsilon=\left\{\varepsilon_{k}\right\}, \varepsilon_{k}=1$ or -1 , and the constant $C$ is independent of $\varepsilon$.

The estimate (2.22) is a consequence of the following:

Lemma 5. Define a sequence $\left\{p_{j}\right\}_{j=1}^{\infty}$ by $p_{1}=2$ and $1 / p_{j+1}=1 / 2+$ $(1-\theta) /\left(2 p_{j}\right)$ for $j \geq 1$. (Note that $1 / p_{j}=\left(1-a^{j}\right) /(1+\theta)$, where $a=(1-\theta) / 2$, so $\left\{p_{j}\right\}$ is decreasing and converges to $1+\theta$.) Then, for $j \geq 1$,

$$
\left\|U_{\varepsilon}^{(m)}(f)\right\|_{p_{j}} \leq C_{j} A B^{2 / p_{j}}\|f\|_{p_{j}} .
$$

Proof. Let

$$
U_{j}^{(m)}(f)=\sum_{k=-\infty}^{\infty} \varepsilon_{k} S_{j+k}\left(\nu_{k}^{(m)} * S_{j+k}(f)\right) .
$$

Then by Plancherel's theorem and the estimates (2.17)-(2.20), as in (2.10) we have

$$
\left\|U_{j}^{(m)}(f)\right\|_{2} \leq C A \min \left(1, \beta_{m}^{-\alpha_{m}(|j|-2)}\right)\|f\|_{2} .
$$

It follows that $\left\|U_{\varepsilon}^{(m)}(f)\right\|_{2} \leq \sum_{j}\left\|U_{j}^{(m)}(f)\right\|_{2} \leq C A B\|f\|_{2}$. If we denote by $A(s)$ the assertion of Lemma 5 for $j=s$, this proves $A(1)$.

Now we derive $A(s+1)$ from $A(s)$, which will complete the proof of Lemma 5 by induction. Using (2.21), we see that

$$
\left(\nu^{(m)}\right)^{*}(f) \leq\left(\mu^{(m)}\right)^{*}(|f|)+\left(\eta^{(m)}\right)^{*}(|f|) \leq g_{m}(|f|)+2\left(\eta^{(m)}\right)^{*}(|f|) .
$$

Note that $A(s)$ implies $\left\|g_{m}(f)\right\|_{p_{s}} \leq C A B^{2 / p_{s}}\|f\|_{p_{s}}$. From this and (2.16) we have

$$
\left\|\left(\nu^{(m)}\right)^{*}(f)\right\|_{p_{s}} \leq\left\|g_{m}(|f|)\right\|_{p_{s}}+2\left\|\left(\eta^{(m)}\right)^{*}(|f|)\right\|_{p_{s}} \leq C A B^{2 / p_{s}}\|f\|_{p_{s}} .
$$

By (2.17), (2.23) and (2.24), we can now apply the arguments used in the proof of (2.15) to get $A(s+1)$. This completes the proof of Lemma 5 .

Now we prove $(2.22)$ for $p \in(1+\theta, 2]$. Let $\left\{p_{j}\right\}_{j=1}^{\infty}$ be as in Lemma 5 . Then we have $p_{N+1}<p \leq p_{N}$ for some $N$. Thus, interpolating between the estimates of Lemma 5 for $j=N$ and $j=N+1$, we have (2.22). This proves (2.3) for $j=m$.

Finally, we can easily see that $\left(\mu^{(0)}\right)^{*}(f) \leq C(\log \varrho)\|\Omega\|_{1}\|h\|_{\Delta_{1}}|f|$ (see (2.17)), which implies the estimate (2.3) for $j=0$. Therefore, by induction we have (2.3) for all $0 \leq j \leq \ell$. This completes the proof of Lemma 1 . 
Now we can prove Theorem 1 . Since $\theta \in(0,1)$ is arbitrary, by taking $\varrho=2^{q^{\prime} s^{\prime}}$ in Lemma 2 we have

$$
\left\|T_{2^{q^{\prime} s^{\prime}}}^{(m)}(f)\right\|_{p} \leq C_{p}(q-1)^{-1}(s-1)^{-1}\|\Omega\|_{q}\|h\|_{\Delta_{s}}\|f\|_{p}
$$

for all $p \in(1, \infty)$. This completes the proof of Theorem 1 , since $T=$ $\sum_{m=1}^{\ell} T_{\varrho}^{(m)}$.

3. Proof of Theorem 2. Theorem 2 can be proved by Theorem 1 and an extrapolation argument. Let $T(f)$ be the singular integral in (1.2). We also write $T(f)=T_{h, \Omega}(f)$. We fix $q \in(1,2], \Omega \in L^{q}\left(S^{n-1}\right), p \in(1, \infty)$ and a function $f$ with $\|f\|_{p} \leq 1$ and put $S(h)=\left\|T_{h, \Omega}(f)\right\|_{p}$. Then we have the subadditivity:

$$
S(h+k) \leq S(h)+S(k) .
$$

Set $E_{1}=\left\{r \in \mathbb{R}_{+}: 0<|h(r)| \leq 2\right\}$ and $E_{m}=\left\{r \in \mathbb{R}_{+}: 2^{m-1}<|h(r)|\right.$ $\left.\leq 2^{m}\right\}$ for $m \geq 2$. Then, applying Theorem 1 , we see that

$$
S\left(h \chi_{E_{m}}\right) \leq C(q-1)^{-1}(s-1)^{-1}\|\Omega\|_{q}\left\|h \chi_{E_{m}}\right\|_{\Delta_{s}}
$$

for $s \in(1,2]$, where $\chi_{E}$ denotes the characteristic function of a set $E$. Now we follow the extrapolation argument of Zygmund [26, Chap. XII, pp. 119-120]. First, note that

$$
\left\|h \chi_{E_{m}}\right\|_{\Delta_{1+1 / m}} \leq 2^{m} d_{m}^{m /(m+1)}(h)
$$

for $m \geq 1$, where $d_{m}(h)$ is as in Section 1. Using this and (3.2) we see that

$$
\begin{aligned}
\sum_{m \geq 1} S\left(h \chi_{E_{m}}\right) & \leq C(q-1)^{-1}\|\Omega\|_{q} \sum_{m \geq 1} m\left\|h \chi_{E_{m}}\right\|_{\Delta_{1+1 / m}} \\
& \leq C(q-1)^{-1}\|\Omega\|_{q} \sum_{m \geq 1} m 2^{m} d_{m}^{m /(m+1)}(h) .
\end{aligned}
$$

Recalling the definition of $N_{a}(h)$, we have

$$
\begin{aligned}
& \sum_{m \geq 1} m 2^{m} d_{m}^{m /(m+1)}(h) \\
& =\sum_{d_{m}(h)<3^{-m}} m 2^{m} d_{m}^{m /(m+1)}(h)+\sum_{d_{m}(h) \geq 3^{-m}} m 2^{m} d_{m}^{m /(m+1)}(h) \\
& \leq \sum_{m \geq 1} m 2^{m} 3^{-m^{2} /(m+1)}+\sum_{m \geq 1} m 2^{m} d_{m}(h) 3^{m /(m+1)} \leq C\left(1+N_{1}(h)\right) .
\end{aligned}
$$

Therefore, by (3.1) we see that

$$
S(h) \leq \sum_{m \geq 1} S\left(h \chi_{E_{m}}\right) \leq C(q-1)^{-1}\|\Omega\|_{q}\left(1+N_{1}(h)\right) .
$$

Next, fix $h \in \mathcal{N}_{1}, p \in(1, \infty)$ and $f$ with $\|f\|_{p} \leq 1$ and let $R(\Omega)=$ $\left\|T_{h, \Omega}(f)\right\|_{p}$. Put $e_{m}=\sigma\left(F_{m}\right)$ for $m \geq 1$, where $F_{m}=\left\{\theta \in S^{n-1}: 2^{m-1}<\right.$ 
$\left.|\Omega(\theta)| \leq 2^{m}\right\}$ for $m \geq 2$ and $F_{1}=\left\{\theta \in S^{n-1}:|\Omega(\theta)| \leq 2\right\}$. We decompose $\Omega$ as $\Omega=\sum_{m=1}^{\infty} \Omega_{m}$, where $\Omega_{m}=\Omega \chi_{F_{m}}-\sigma\left(S^{n-1}\right)^{-1} \int_{F_{m}} \Omega d \sigma$. We note that $\int \Omega_{m} d \sigma=0,\left\|\Omega_{m}\right\|_{r} \leq C 2^{m} e_{m}^{1 / r}$ for $1<r<\infty$. Now, by (3.3) and the subadditivity of $R(\Omega)$ we see that

$$
\begin{aligned}
R(\Omega) & \leq \sum_{m \geq 1} R\left(\Omega_{m}\right) \leq C\left(1+N_{1}(h)\right) \sum_{m \geq 1} m\left\|\Omega_{m}\right\|_{1+1 / m} \\
& \leq C\left(1+N_{1}(h)\right) \sum_{m \geq 1} m 2^{m} e_{m}^{m /(m+1)} \\
& =C\left(1+N_{1}(h)\right)\left(\sum_{e_{m}<3^{-m}}+\sum_{e_{m} \geq 3^{-m}}\right) \\
& \leq C\left(1+N_{1}(h)\right)\left(\sum_{m \geq 1} m 2^{m} 3^{-m^{2} /(m+1)}+\sum_{m \geq 1} m 2^{m} e_{m} 3^{m /(m+1)}\right) \\
& \leq C\left(1+N_{1}(h)\right)\left(1+\int_{S^{n-1}}|\Omega(\theta)| \log (2+|\Omega(\theta)|) d \sigma(\theta)\right) .
\end{aligned}
$$

This completes the proof of Theorem 2 .

4. Estimates for maximal functions. For the maximal operator $T^{*}$ in (1.3) we have a result similar to Theorem 1.

Theorem 3. Let $q \in(1,2], s \in(1,2]$ and $\Omega \in L^{q}\left(S^{n-1}\right), h \in \Delta_{s}$. Suppose $\Omega$ satisfies (1.1). Then

$$
\left\|T^{*}(f)\right\|_{L^{p}\left(\mathbb{R}^{d}\right)} \leq C_{p}(q-1)^{-1}(s-1)^{-1}\|\Omega\|_{L^{q}\left(S^{n-1}\right)}\|h\|_{\Delta_{s}}\|f\|_{L^{p}\left(\mathbb{R}^{d}\right)}
$$

for all $p \in(1, \infty)$, where $C_{p}$ is independent of $q, s, \Omega$ and $h$.

As Theorem 1 implies Theorem 2, we have the following theorem as a consequence of Theorem 3 .

THEOREM 4. Let $\Omega$ be a function in $L \log L\left(S^{n-1}\right)$ satisfying (1.1) and $h \in \mathcal{N}_{1}$. Then

$$
\left\|T^{*}(f)\right\|_{L^{p}\left(\mathbb{R}^{d}\right)} \leq C_{p}\|f\|_{L^{p}\left(\mathbb{R}^{d}\right)}
$$

for all $p \in(1, \infty)$.

As in the cases of Theorems 1 and 2, the constants $C_{p}$ of Theorems 3 and 4 are also independent of the polynomials $P_{j}$ if we fix $\operatorname{deg}\left(P_{j}\right)(j=1, \ldots, d)$. When $\Omega$ is as in Theorem 4 and $h \in \Delta_{s}$ for some $s>1, L^{p}$ boundedness of $T^{*}$ was proved in [1]. When $n=d, P(y)=y, \Omega \in L^{q}$ for some $q>1$ and $h$ is bounded, $L^{p}$ boundedness of $T^{*}$ is due to [3].

We use the following result to prove Theorem 3 .

Lemma 6. Let $\tau^{(m)}=\left\{\tau_{k}^{(m)}\right\}(1 \leq m \leq \ell)$, where the measures $\tau_{k}^{(m)}$ are as in $(2.2)$. Let $\theta \in(0,1)$ and let positive numbers $A=(\log \varrho)\|\Omega\|_{L^{q}\left(S^{n-1}\right)}\|h\|_{\Delta_{s}}$, 
$B=\left(1-\beta_{m}^{-\theta \alpha_{m}}\right)^{-1}$ be as above. Define

$$
T_{\varrho, m}^{*}(f)(x)=\sup _{k \in \mathbb{Z}}\left|\sum_{j=k}^{\infty} \tau_{j}^{(m)} * f(x)\right| .
$$

Then for $p \in\left(2(1+\theta) /\left(\theta^{2}-\theta+2\right),(1+\theta) / \theta\right)=: I_{\theta}$ we have

$$
\left\|T_{\varrho, m}^{*}(f)\right\|_{p} \leq C A\left(B^{1+\delta(p)}+B^{2 / p+1-\theta / 2}\right)\|f\|_{p},
$$

where $C$ is independent of $q, s \in(1,2], \Omega \in L^{q}\left(S^{n-1}\right), h \in \Delta_{s}$, @ and the coefficients of the polynomials $P_{j}(1 \leq j \leq d)$.

Proof. Let $T_{\varrho}^{(m)}(f)=\sum_{k} \tau_{k}^{(m)} * f$ be as in Lemma 2. Let a function $\varphi$ be as in the definition of $\tau_{k}^{(m)}$ in $(2.2)$. Define $\varphi_{k}$ by $\hat{\varphi}_{k}(\xi)=$ $\varphi\left(\beta_{m}^{k}\left|H_{m} \pi_{s_{m}}^{d} R_{m}(\xi)\right|\right)$. Let $\delta$ be the delta function as above. Following [8], we decompose

$\sum_{j=k}^{\infty} \tau_{j}^{(m)} * f=\varphi_{k} * T_{\varrho}^{(m)}(f)-\varphi_{k} *\left(\sum_{j=-\infty}^{k-1} \tau_{j}^{(m)} * f\right)+\left(\delta-\varphi_{k}\right) *\left(\sum_{j=k}^{\infty} \tau_{j}^{(m)} * f\right)$.

It follows that

$$
T_{\varrho, m}^{*}(f) \leq \sup _{k}\left|\varphi_{k} * T_{\varrho}^{(m)}(f)\right|+\sum_{j=0}^{\infty} N_{j}^{(m)}(f),
$$

where $N_{j}^{(m)}(f)=\sup _{k}\left|\varphi_{k} *\left(\tau_{k-j-1}^{(m)} * f\right)\right|+\sup _{k}\left|\left(\delta-\varphi_{k}\right) *\left(\tau_{j+k}^{(m)} * f\right)\right|$. By Lemma 2 we have

$$
\left\|\sup _{k}\left|\varphi_{k} * T_{\varrho}^{(m)}(f)\right|\right\|_{p} \leq C A B^{1+\delta(p)}\|f\|_{p} \quad \text { for } p \in(1+\theta,(1+\theta) / \theta) .
$$

Also, by (2.7) we see that

$$
\left\|N_{j}^{(m)}(f)\right\|_{r} \leq C A B^{2 / r}\|f\|_{r} \quad \text { for } r>1+\theta .
$$

On the other hand, we have

$$
N_{j}^{(m)}(f) \leq\left(\sum_{k}\left|\left(\delta-\varphi_{k}\right) *\left(\tau_{j+k}^{(m)} * f\right)\right|^{2}\right)^{1 / 2}+\left(\sum_{k}\left|\varphi_{k} *\left(\tau_{k-j-1}^{(m)} * f\right)\right|^{2}\right)^{1 / 2} .
$$

Therefore, by the estimates (2.5), (2.6) and Plancherel's theorem, we get

$$
\left\|N_{j}^{(m)}(f)\right\|_{2} \leq C A \beta_{m}^{-\alpha_{m} j}\left(1-\beta_{m}^{-2 \alpha_{m}}\right)^{-1 / 2}\|f\|_{2}
$$

(see $\left[8\right.$, p. 820]). For $p \in I_{\theta}$ we can find $r \in(1+\theta, 2(1+\theta) / \theta)$ such that $1 / p=(1-\theta) / r+\theta / 2$, so interpolation between (4.4) and (4.5) implies that

$$
\left\|N_{j}^{(m)}(f)\right\|_{p} \leq C A B^{2(1-\theta) / r}\left(1-\beta_{m}^{-2 \alpha_{m}}\right)^{-\theta / 2} \beta_{m}^{-\alpha_{m} \theta j}\|f\|_{p} .
$$

Therefore, by (4.2), (4.3) and (4.6), for $p \in I_{\theta}$ we have

$$
\left\|T_{\varrho, m}^{*}(f)\right\|_{p} \leq C A\left(B^{1+\delta(p)}+B^{2(1-\theta) / r+1}\left(1-\beta_{m}^{-2 \alpha_{m}}\right)^{-\theta / 2}\right)\|f\|_{p} .
$$


This implies the conclusion of Lemma 6 , since $\left(1-\beta_{m}^{-2 \alpha_{m}}\right)^{-1} \leq B$ and $2(1-\theta) / r+\theta / 2+1=2 / p+1-\theta / 2$.

Proof of Theorem 3. Note that $T^{*}(f) \leq 2 T_{0}^{*}(f)+2 \mu_{\varrho}^{*}(|f|)$, where $T_{0}^{*}(f)$ is defined by the formula in (4.1) with $\left\{\tau_{j}^{(m)}\right\}$ replaced by the sequence $\left\{\sigma_{j}\right\}$ of measures in $(2.1)$, and $\mu_{\varrho}^{*}=\left(\mu^{(\ell)}\right)^{*}$ is as in Lemma 1. We note that $T_{0}^{*}(f) \leq \sum_{m=1}^{\ell} T_{\varrho, m}^{*}(f)$. Now, Lemma 6 implies that

$$
\left\|T_{\varrho, m}^{*}(f)\right\|_{p} \leq C(\log \varrho)\left(1-\varrho^{-\theta /\left(2 q^{\prime} s^{\prime}\right)}\right)^{-3}\|\Omega\|_{q}\|h\|_{\Delta_{s}}\|f\|_{p}
$$

for $p \in I_{\theta}$. By using this with $\varrho=2^{q^{\prime} s^{\prime}}$, since $\theta \in(0,1)$ is arbitrary, we can conclude that

$$
\left\|T_{2^{q^{\prime} s^{\prime}, m}}^{*}(f)\right\|_{p} \leq C_{p}(q-1)^{-1}(s-1)^{-1}\|\Omega\|_{q}\|h\|_{\Delta_{s}}\|f\|_{p}
$$

for $p \in(1, \infty)$. Also, by Lemma $1, \mu_{\varrho}^{*}$ satisfies a similar estimate when $\varrho=2^{q^{\prime} s^{\prime}}$. Collecting results, we have Theorem 3 .

REMARK. Let

$$
M(f)(x)=\sup _{t>0} t^{-n} \int_{|y|<t}|f(x-P(y))|\left|\Omega\left(y^{\prime}\right)\right||h(|y|)| d y .
$$

It is easy to see that $M(f) \leq C \mu_{\varrho}^{*}(f)$, where $C$ is independent of $\varrho \geq 2$. Therefore, by Lemma 1 we can prove results similar to Theorems 1 and 2 for the maximal operator $M$. In [1], $L^{p}$ boundedness of $M$ was proved under the condition that $\Omega \in L \log L\left(S^{n-1}\right)$ and $h \in \Delta_{s}$ for some $s>1$. When $n=d$, $P(y)=y$, it is known that $M$ is of weak type $(1,1)$ if $\Omega \in L \log L\left(S^{n-1}\right)$ and $h$ is bounded (see $[5,4]$ ).

\section{References}

[1] A. Al-Salman and Y. Pan, Singular integrals with rough kernels in $L \log L\left(S^{n-1}\right)$, J. London Math. Soc. (2) 66 (2002), 153-174.

[2] A. P. Calderón and A. Zygmund, On singular integrals, Amer. J. Math. 78 (1956), 289-309.

[3] L. K. Chen, On a singular integral, Studia Math. 85 (1987), 61-72.

[4] M. Christ, Weak type $(1,1)$ bounds for rough operators, Ann. of Math. 128 (1988), $19-42$.

[5] M. Christ and J. L. Rubio de Francia, Weak type $(1,1)$ bounds for rough operators, II, Invent. Math. 93 (1988), 225-237.

[6] J. Duoandikoetxea, Weighted norm inequalities for homogeneous singular integrals, Trans. Amer. Math. Soc. 336 (1993), 869-880.

[7] J. Duoandikoetxea and J. L. Rubio de Francia, Maximal and singular integral operators via Fourier transform estimates, Invent. Math. 84 (1986), 541-561.

[8] D. Fan and Y. Pan, Singular integral operators with rough kernels supported by subvarieties, Amer. J. Math. 119 (1997), 799-839. 
[9] D. Fan and S. Sato, Weighted weak type $(1,1)$ estimates for singular integrals and Littlewood-Paley functions, Studia Math. 163 (2004), 119-136.

[10] R. Fefferman, A note on singular integrals, Proc. Amer. Math. Soc. 74 (1979), 266-270.

[11] S. Hofmann, Weak $(1,1)$ boundedness of singular integrals with nonsmooth kernel, ibid. 103 (1988), 260-264.

[12] -, Weighted weak-type $(1,1)$ inequalities for rough operators, Proc. Amer. Math. Soc. 107 (1989), 423-435.

[13] J. Namazi, On a singular integral, ibid. 96 (1986), 421-424.

[14] F. Ricci and E. M. Stein, Harmonic analysis on nilpotent groups and singular integrals, I, J. Funct. Anal. 73 (1987), 179-194.

[15] S. Sato, Estimates for singular integrals along surfaces of revolution, J. Austral. Math. Soc., to appear.

[16] —, Estimates for singular integrals associated with manifolds of finite type and extrapolation, preprint.

[17] -, Estimates for Littlewood-Paley functions and extrapolation, Integral Equations Oper. Theory 62 (2008), 429-440.

[18] A. Seeger, Singular integral operators with rough convolution kernels, J. Amer. Math. Soc. 9 (1996), 95-105.

[19] A. Seeger and T. Tao, Sharp Lorentz space estimates for rough operators, Math. Ann. 320 (2001), 381-415.

[20] E. M. Stein, Problems in harmonic analysis related to curvature and oscillatory integrals, in: Proc. Int. Congress of Mathematicians, Berkeley, 1986, 196-221.

[21] —, Harmonic Analysis: Real-Variable Methods, Orthogonality, and Oscillatory Integrals, Princeton Univ. Press, Princeton, NJ, 1993.

[22] E. M. Stein and G. Weiss, Introduction to Fourier Analysis on Euclidean Spaces, Princeton Univ. Press, Princeton, NJ, 1971.

[23] T. Tao, The weak-type $(1,1)$ of $L \log L$ homogeneous convolution operator, Indiana Univ. Math. J. 48 (1999), 1547-1584.

[24] A. Vargas, Weighted weak type $(1,1)$ bounds for rough operators, J. London Math. Soc. (2) 54 (1996), 297-310.

[25] D. Watson, Weighted estimates for singular integrals via Fourier transform estimates, Duke Math. J. 60 (1990), 389-399.

[26] A. Zygmund, Trigonometric Series, 2nd ed., Cambridge Univ. Press, Cambridge, London, 1977.

Department of Mathematics

Faculty of Education

Kanazawa University

Kanazawa 920-1192, Japan

E-mail: shuichi@kenroku.kanazawa-u.ac.jp 